\title{
Stable and precise optical bench for space applications
}

Giannas Drougkakis, Vaggelis Tzardis, Debapriya Pal, Vinay Pareek, Georgios Vasilakas, et al.

Giannas Drougkakis, Vaggelis Tzardis, Debapriya Pal, Vinay Pareek, Georgios Vasilakas, Nikolaos Papadakis, Dimitrios G. Papazoglou, Wolf von Klitzing, "Stable and precise optical bench for space applications," Proc. SPIE 11852, International Conference on Space Optics - ICSO 2020, 118526R (11 June 2021); doi: 10.1117/12.2600319

SPIE Event: International Conference on Space Optics - ICSO 2021, 2021, Online Only 


\section{International Conference on Space Optics-ICSO 2020}

Virtual Conference

30 March-2 April 2021

Edited by Bruno Cugny, Zoran Sodnik, and Nikos Karafolas
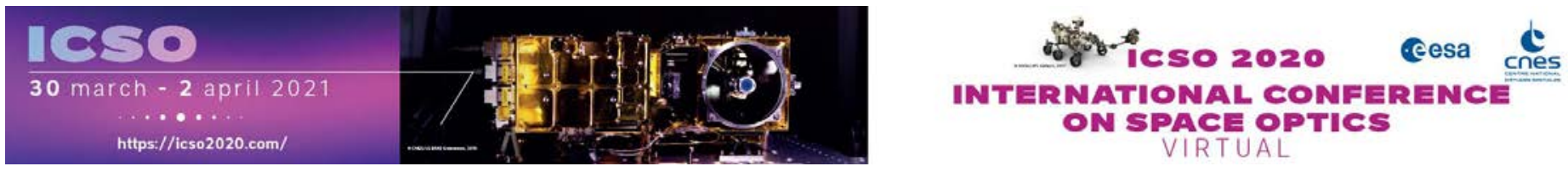

\section{Stable and precise optical bench for space applications}

\section{Cesa isopmeatians ecnes}




\title{
Stable and precise optical bench for space applications
}

\author{
Giannis Drougakis ${ }^{\mathrm{a}, \mathrm{b}}$, Vaggelis Tzardis ${ }^{\mathrm{a}}$, Debapriya Pal ${ }^{\mathrm{a}}$, Vinay Pareek ${ }^{\mathrm{a}, \mathrm{b}}$, Georgios Vasilakis $^{\mathrm{a}}$, \\ Nikolaos Papadakis ${ }^{c}$, Dimitrios G. Papazoglou ${ }^{\mathrm{a}, \mathrm{b}}$, and Wolf von Klitzing ${ }^{\mathrm{a}}$ \\ ${ }^{a}$ Institute of Electronic Structure and Laser, Foundation for Research and Technology-Hellas, \\ 70013 Heraklion, Greece \\ ${ }^{\mathrm{b}}$ Department of Materials Science and Technology, University of Crete, 70013 Heraklion, \\ Greece \\ Department of Mechanical Engineering, Hellenic Mediterranean University, 71410 Heraklion, \\ Greece
}

\begin{abstract}
We report a novel Optical Beam Steering Technique (OBST) for fiber to free-space to fiber coupling with extreme stability of the coupling efficiency and high tolerance to temperature fluctuations. The compact optical system is based near monolithic (ZERODUR) fiber couplers and relies on optical wedges and plates to perform the fine-steering of the optical beam. In our approach, we use coarsely aligned fiber couplers and then perform the fine alignment using the wedges and plates. This separation of fine and course tuning significantly reduces the manufacturing and assembly requirements of the fiber couplers. At the same time, it results in a much-reduced sensitivity to drifts increases the stability and reliability of the breadboard. OBST achieves a coupling efficiency (CE) of $94 \%$ with long-term fluctuation of $0.1 \%$ RMS at stable temperatures and $1.4 \%$ under repeated vacuum temperature cycling over a $30 \mathrm{~K}$ range. Even under extreme mechanical stress (random vibrations of up to 8.3g) the RMS vibration of the coupling efficiency were less than $0.3 \%$ RMS. The optical breadboard technology has therefore achieved TRL5.
\end{abstract}

Keywords: Beam steering, Chemical species, Fiber couplers, Glasses, Optical alignment, Optical components, Optics manufacturing, Temperature metrology, Transmitters, Zerodur

\section{INTRODUCTION}

With the arrival on satellites of LIDAR, optical communication, laser ranging, and quantum technologies, the complexity of optical experiments in space has increased enormously. High-capacity communication links between ground stations and satellites are crucial for many applications, such as earth observation, due to the large information capacity required. ${ }^{1}$ In order to achieve the high sensitivities needed, optical fiber amplifiers can be used where the light is coupled into single-mode fibers. ${ }^{2}$ In addition, to avoid cloud coverage, the optical signal from multiple optical ground stations needs to be simultaneously coupled to several optical fibers on the space terminal. This increases the complexity of the system and the need for stable optical beam steering technology. Quantum sensors and atom clocks, on the other hand, rely their operation on complex light-conditioning breadboards. A large number of proposed missions incorporates these devices, aiming to perform experiments in the context of dark matter search and gravitational-wave astronomy, ${ }^{3}$ gravity sensing and fundamental physics. ${ }^{4,5}$

In many cases, the light-conditioning can be handled by in-fibre devices; however, often the requirements exceed what can be achieved in single mode waveguides. For instance, if frequency shifting realized with acoustooptic modulators or very high extinction ratios are needed, the light has to be coupled out of the fiber onto a freespace breadboard and back into the fiber. The fiber's single mode nature results in very stringent requirements for the optical breadboard and its components. The PHARAO mission was forced to use active stabilization of many optical components increasing the risk of failure. ${ }^{6}$ For LISA-Pathfinder a breadboard optical bonding was chosen, where alignment errors have to be corrected by re-machining the optical components. ${ }^{7}$ Clearly, these

Further author information: (Send correspondence to Wolf von Klitzing.)

Wolf von Klitzing.: E-mail: wvk@iesl.forth.gr, Telephone: 00302810391544 
solutions are very costly and/or complex to become common practice in space missions. On another approach optical wedges were used to couple light into single-mode fibers. ${ }^{8}$ This technology was applied in space atom clock in 2018. ${ }^{9}$ According to the authors, the results indicate that the optical system is suitably robust for the space applications for which the rubidium atomic clock was built, but this high stability occurs only within a limited temperature range.

In this paper, we report on the developments of a novel beam steering technique based on the use of optical wedges and flats to perform the steering of the beam in a precise and robust fashion. Compared to our first prototype,${ }^{10}$ we achieved to reduce the optical breadboard's volume by $54 \%$. The performance of the breadboard has been demonstrated in the presence of $30 \mathrm{~K}$ temperature fluctuations under vacuum and up to $8 \mathrm{~g}$ vibrations in atmospheric pressure.

\section{DESIGN FEATURES}

One of the objectives of the proposed technique was to reduce the overall cost of optical breadboards by a) optimizing the size of the coupler/collimator b) using a material that exhibits a lower coefficient of thermal expansion (CTE) c) miniaturizing the optical components. The performance of a prototype breadboard was demonstrated under a relevant to the applications identified environment. The material of the breadboard components was selected to be the ZERODUR DK0 class, with less than half the CTE of the DK1 class used in our previous approach. In addition The volume of all the breadboard components was reduced by $54 \%$ compared to the previous approach. We performed a finite element thermomechanical analysis to understand the effect of temperature variation on the ZERODUR components in order to mimic the possible scenarios inside a space vehicle. The analysis showed that ZERODUR components reveal minimal mechanical deformation for the critical optomechanical interfaces such as lens-coupler and fiber-coupler interfaces. This deformation is in the nanometers and micro degrees range.

The breadboard was inserted into a specially designed vacuum chamber. On the surface of the latter, the temperature varied in the range $[10,40]^{\circ} \mathrm{C}$ with a PID-controlled heating/cooling rate equal to $10^{\circ} \mathrm{C} / \mathrm{h}$. The coupling efficiency (CE) of the breadboard was measured to reach $94.4 \%$. In addition, the CE fluctuations under stable temperature was measured to be $0.1 \%$ RMS, while under thermal cycling $1.36 \%$. As for the mechanical durability tests, vibration tests were performed according to rigorous standards (see Table1) with the acceleration reaching $3.5 \mathrm{~g}$ in sweep mode, $8.3 \mathrm{~g}$ in random accelerations and $89.4 \mathrm{~g}$ for $1 \mathrm{~ms}$ in shock testing.

\section{THERMOMECHANICAL ANALYSIS}

We performed an in depth analysis of the system's behaviour in the presence of temperature variations. The setup and workflow of the finite element simulation is presented below. We examine the steady-state thermal analysis over a range of temperatures and discuss the effects of thermal deformation results on coupling efficiency. The investigation included three different levels of the system, namely the a) Breadboard, b) the Mount-Wedge holder - prism and, c) the Coupler lens system. For each system, the effect of the temperature differential was investigated. We find that the displacement due to exposure to a temperature gradient and the resulting changes in coupling efficiency is practically negligible.

All the calculations were performed in SolidWorks. In order to calculate the deformation two separate simulations are carried out. Initially, a thermal analysis which calculates the thermal profile for the system under investigation and, subsequenctly a structural analysis is performed using the aforementioned thermal profile which calculates the deformation (among other quantities).

\subsection{Breadboard Thermomechanical analysis}

\section{Boundary conditions for the Thermal simulation}

In order to simulate the thermomechanical behaviour of the breadboard in space, the following setup was used. The breadboard was enclosed inside a metallic enclosure and rested on 4 mounting feet. The feet were made from an insulating material. Complete vacuum is assumed therefore there is no convective heat transfer. 
Table 1: Specification of OBST prototype

- Extremely high coupling efficiency of $94.4 \%$

- Exceedingly small fluctuation of the coupling efficiency

under extreme (relevant) conditions

- Stable temperature: $<0.1 \%_{R M S} @ 25.5^{\circ} \mathrm{C}$ over a period of 5 days

- Thermal Cycling: <1.4\%RMS during $\sim 6$ days of thermal cycling between 10 and $40^{\circ} \mathrm{C} @ 10.05 K / h$

- Vibration < $1.4 \%_{R M S} @ 8.3 \mathrm{~g}$

- Vibration testing was successful and the breadboard complied

to requirements of the following standards:

- EN 60068-2-6:2008 (IEC 60068-2-6:2007)

- EN 60068-2-64:2008 (IEC 60068-2-64:2008)

- ISO 20780:2018

- The couplers have been miniaturized by a factor 2 in volume compared to our previous work. ${ }^{10}$

Conductive heat transfer is allowed to occur in all body surfaces that are in contact with other bodies. These surfaces are assumed bonded.

Radiative heat transfer was simulated with the use of the following thermal boundary conditions. For the enclosure, the following Dirichlet boundary conditions were set: the Internal Bottom surface (where the feet are attached) was set to $23^{\circ} \mathrm{C}$ and the Internal Top surface was set to $33^{\circ} \mathrm{C}$. This achieved a temperature difference of 10K. By adjusting the Dirichlet BCs it was possible to test at different temperature differences. The temperature differences that were investigated were $10 \mathrm{~K}, 20 \mathrm{~K}$ and $40 \mathrm{~K}$. For the remaining internal surfaces of the enclosure box Von Neuman Boundary conditions were used.

\section{Boundary conditions for the structural simulation}

The mechanical boundary conditions were set such as to fix one point in space and allow the remaining structure to deform freely without artificial constraints. Additionally, in the structural simulation the box and the mounting tabs are excluded from the analysis (the elasticity modulus of the mounting tabs is so small that essentially the BB and the mechanical box are uncoupled and their inclusion only increased simulation processing time without improving the accuracy).

The thermal and structural analysis parameters previously described constitute a simple model that imitates a test setup. The thermal profile is shown in Fig. 1 while an example of typical deformation can be seen in Fig. 2 . 




Figure 1: Temperature profile. The top and bottom of the enclosure are set to $43^{\circ} \mathrm{C}$ and $23^{\circ} \mathrm{C}$ respectively. The temperature difference between the top and bottom at the center of the BB is only $4.7 \mathrm{~K}$

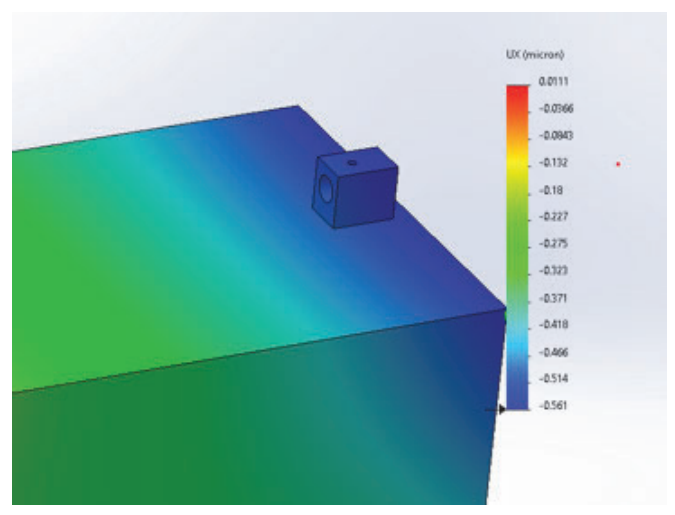

a

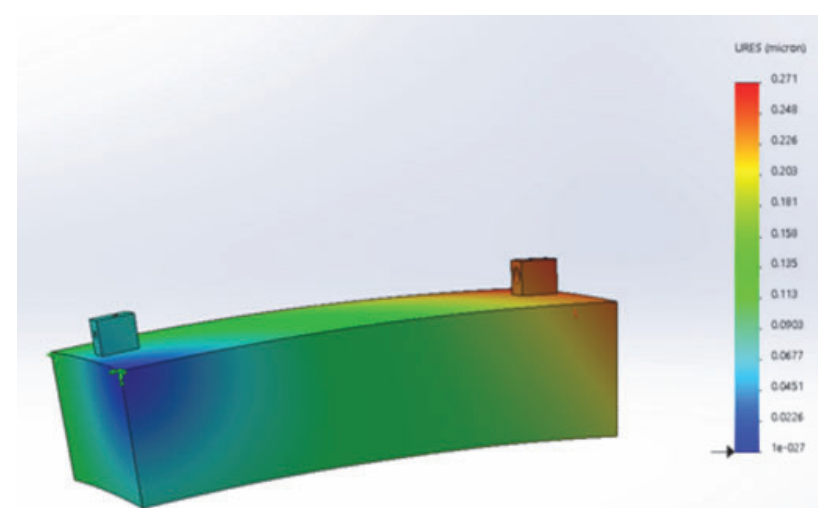

b

Figure 2: Thermal and structural analysis results for the optical breadboard. a)Resultant deformation with the thermal analysis, b)Total resultant displacement (Exaggeration of the deformed state (150x) for visual purposes

\subsection{Mount-Wedge holder-Prism system Thermomechanical Analysis}

The aim of the simulation was to determine if the different thermal expansion of the UV fused silica wedge and the Zerodur wedge holder would result in considerable strains on the wedge. Increased strain might affect the optical properties of the wedge. All surfaces between different parts are assumed bonded where any faces are touching. The fixing points used were the points of the wedge holder-mount interface. For the thermal load, a constant temperature of $100^{\circ} \mathrm{C}$, significantly higher compared to the expected specifications, was set throughout the system. The temperature for zero deformation was set at $23^{\circ} \mathrm{C}$. The simulation performed of the entire system (wedge holder-wedge) revealed minimal strain (5.35e-5) as shown in Fig.3.

\subsection{Coupler Lens system Thermomechanical Analysis}

The aim of this analysis was to determine what is the maximum displacement observed on the lens when it is fitted in the coupler. In the main breadboard analysis, the lens was not included. The tradeoff to include the lens in the analysis was not favorable (significantly increased model complexity and processing time for a small effect). As a compromise, it was considered a better solution to investigate the effect of the lens displacement in the coupler in a submodel i.e. the Coupler - Lens system.

For the thermal load, a temperature of $23^{\circ} \mathrm{C}$ and $33^{\circ} \mathrm{C}$ was set on the bottom and top surface. The temperature for zero deformation was set at $23^{\circ} \mathrm{C}$. For this simulation, the fixing points used were the points of the center of the lens, in order to be able to estimate the displacement of the lens. The layer of adhesives was not included in the model. The reasoning behind this approach is that the glue is much thinner than $100 \mu \mathrm{m}$. 


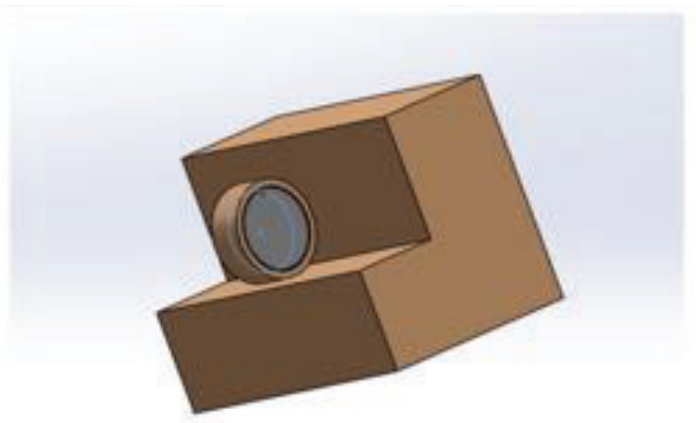

a) b)

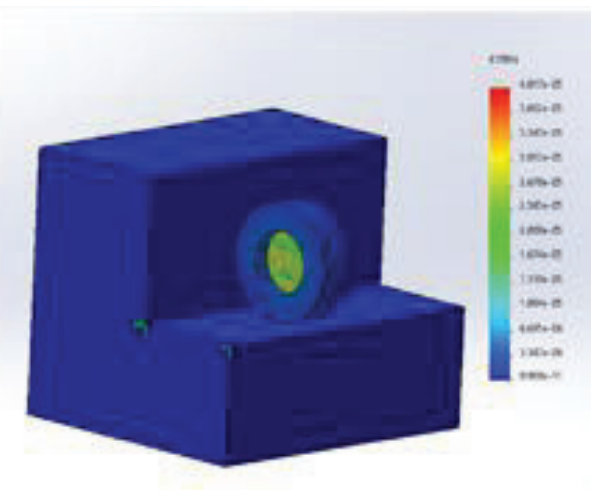

Figure 3

Mechanical layout and structural analysis results for the wedge holder-wedge system. a)Wedge holder including UV fused silica prism and ZERODUR mounting blocks for thermal/displacement/strain analysis, b)Resultant strain of the mount-wedge holder-prism system

As a result any expansion in it's thickness even for a temperature difference of more than $10 \mathrm{~K}$ is bound to be submicron. Additionally, a significant characteristic which can contribute to the deformation of the adhesive(i.e. the non uniform thickness) cannot be accurately simulated. Fig. 4 presents the coupler's structural analysis data in terms of radial displacement wrt the symmetry axis of the lens, and it can be seen that the displacement is in the order of 0.02 microns.

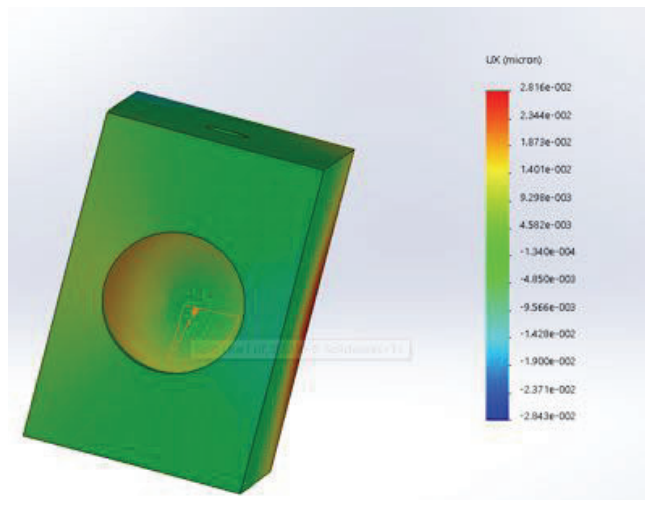

Figure 4: Resultant Radial displacement component of the Coupler lens system

\subsection{Conclusions of thermal simulation}

The thermal simulation of the ZERODUR components revealed minimal mechanical deformation for the critical optomechanical interfaces. This deformation was in the $\mathrm{nm}$ and micro degree range for the temperature range the thermal testing was performed. This analysis took into account only the displacement of the optical elements (lenses, fiber ferrule) and not their differential expansion. The thermal simulation of the wedge holder with the prism mounted as well as the simulation of the prism at $77 K$ temperature difference only showed mechanical compressive strains in the order of $0.003 \%$. The coupler lens system simulation at temperature difference $10 K$ (top and bottom surface) also exhibited sub micron displacements. Overall, the simulation results suggested that the thermomechanical deformation of the ZERODUR components is in the order of submicron. 


\section{OPTOMECHANICAL DESIGN}

OBST design inherits a large number of the critical features developed in the context of our previous work. ${ }^{10}$ Nevertheless, significant improvement has been achieved such as a notable decrease in volume by $54 \%$ and increased stability due to the different approach in the mounting of the optical fiber into the fiber coupler. All the mechanical mounts for the optical components can be seen in Fig. 5.

One point of emphasis of the OBST design is the reduction in complexity of the fiber coupler as in Fig. 5a, which results in simpler manufacturing, higher precision, larger stability, and easier assembly. It is a ZERODUR block to which we drill three concentric holes with different diameters. This nearly monolithic design greatly simplifies the manufacturing and assembly of the coupler on the breadboard, whilst improving its stability.

The most important factor for the stability of the OBST breadboard is the concentricity of the fiber and the lens. A vertical displacement of the fiber by only a few micrometers will cause a total loss of coupling. One of the essential aspects of OBST is to keep the construction of the coupler as simple as possible with as few adjustable parts as possible. The ferrule of the optical fiber is inserted into a glass sleeve, which in turn is inserted into the zerodur coupler. The tight tolerances of the components together with the cylindrical symmetry of the bonding surfaces ensures maximum stability against thermal expansion or mechanical misalignments.

The lens is mounted into a counterbore. The concentricity of the counterbore is ensured by using a lathe that drills consecutively the hole for the placement of the lens and for the fiber. The size of the counterbore is chosen small enough so that the lens is sufficiently constrained in position for the OBST wedge and plate system to compensate any misalignment in angle. It is chosen large enough to accommodate the tolerances in the sizes of the hole and lens, leaving sufficient space so that the lens does not touch the sides of the counterbore. The lens is bonded to the $2 \mathrm{~mm}$ thick rim of the counterbore (rather than its sides), thus ensuring that the differential thermal expansion does not put undue pressure on the lens.

a)

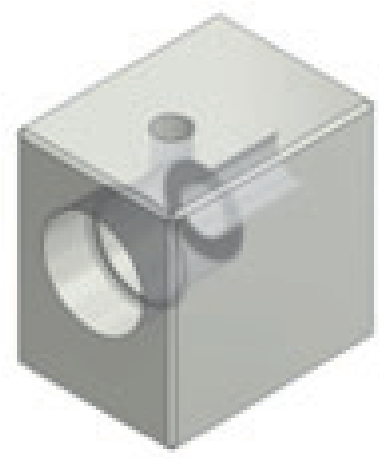

b)

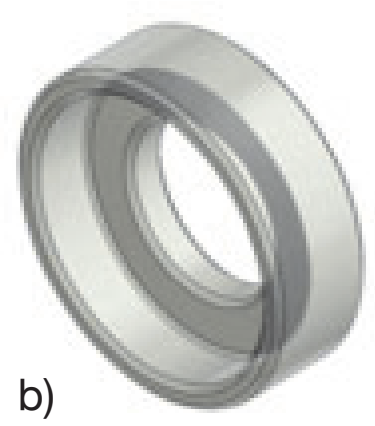

c)

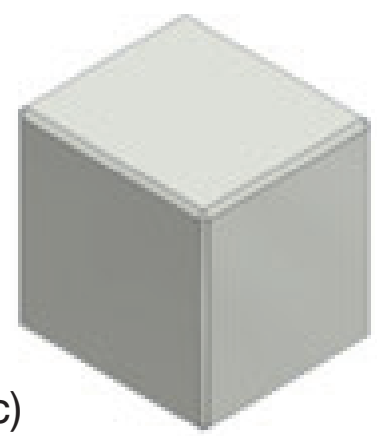

Figure 5: ZERODUR components a) fiber coupler, b) wedge holder, c) supporting cube

The optical fiber is terminated on one side by an AR-coated FC-APC fiber connector, and on the other end by an AR coated APC ceramic ferrule of $1.8 \mathrm{~mm}$ diameter. The ferrule is inserted into a glass sleeve as seen in Fig. 6 (note that the tolerances are only $\pm 5 \mu \mathrm{m}$ for the ferrule and $\pm 0.010 \mathrm{~mm}$ for the sleeve, thus ensuring near-perfect fit). The sleeve is then inserted into the ZERODUR coupler block hole.

After validating the compliance of the received optomechanical components with the specifications, the fiber to fiber alignment was performed in the following steps. First the collimator was assembled by bonding the lens into the block, inserting the fiber-ferrule and collimating the beam. The collimation is monitored and recorded using a novel and precise technique ${ }^{11}$ that was developed in our group in the context of OBST. Our method quickly and accurately measures the diameters of Gaussian, Airy or Bessel beams ranging from submillimeter to many centimeters without specialized equipment. The ferrule was then fixed in place by UV-curing the adhesive. Second, the collimated fiber couplers were bonded onto the breadboard after a rough alignment. Then, the corrective optics (optical wedge pair and plate) are put in place and aligned using standard laboratory translation stages and mirror mounts. The $\mathrm{CE}$ was continuously monitored using a photodiode. Finally, after 


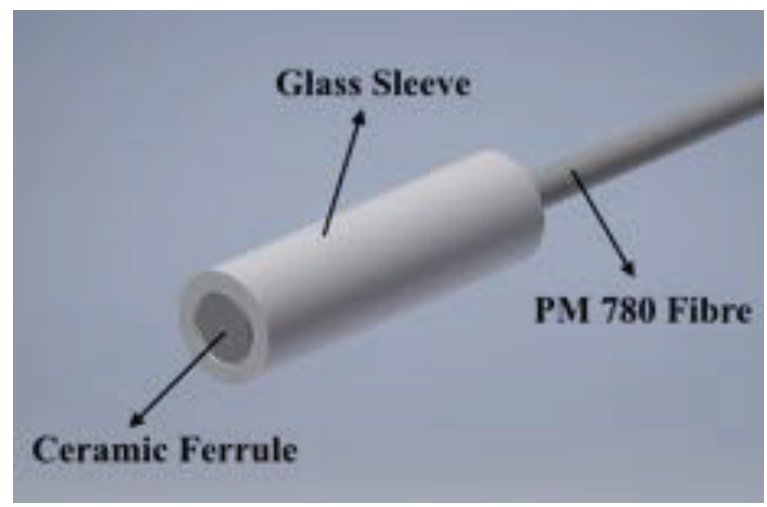

Figure 6: Ferrule inside the glass sleeve.

the maximum $\mathrm{CE}$ was achieved, all the components were fixed in place by UV-curing a thin layer of glue that was applied on the components before the start of the process. The integrated OBST breadboard can be seen in Fig. 7.

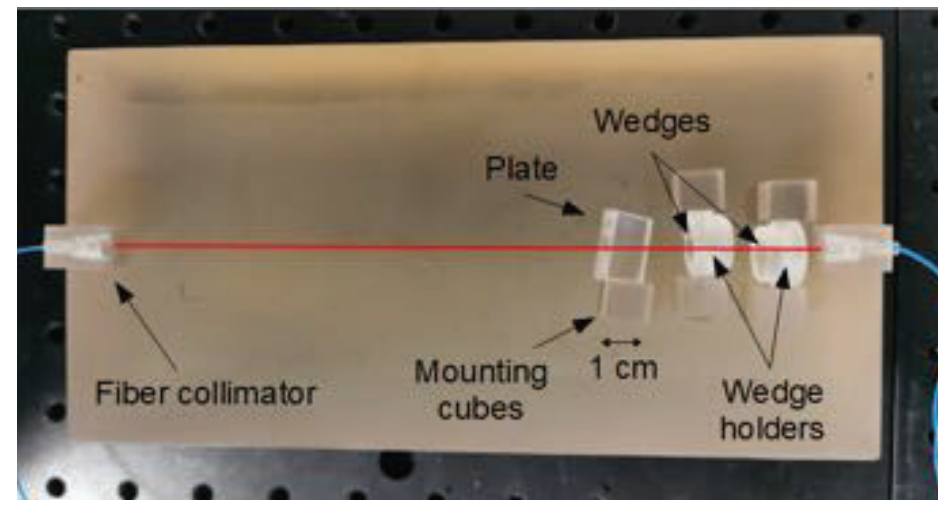

Figure 7: The integrated OBST breadboard. The red line represents the optical path.

\section{TEST RESULTS}

The performance of the breadboard was evaluated in the presence of temperature fluctuation under vacuum and under vibration in atmospheric pressure. For the thermal tests, in order to avoid thermal effects of the responsivity of the photodiode we measured both the input and output light on the breadboard inside the vacuum chamber using the same photodiode. That was achieved by putting the breadboard in a metallic structure with a mounted servo motor - photodiode system that moved repeatedly between the measurement point of the input and output light. All of these components were placed inside a vacuum chamber $\left(P=10^{-3}\right.$ Torr $)$. The fiber, the thermocouples, the vacuum sensor, the servo power/signal and the photodiode readings were connected using vacuum feedthrough connectors (Fig.8a).

The breadboard was thermally tested under stable $\left(25.5^{\circ} \mathrm{C}\right)$ and ramping temperature (range on the walls of the vacuum chamber: $[10,40]^{\circ} \mathrm{C}$, rate: $10^{\circ} \mathrm{C} / \mathrm{h}$ ). For this purpose, the system was cooled using a commercial freezer and heated with heating tapes along with a PID controller for linear temperature ramping (see Fig.8b). The chamber was placed inside a thermally insulating foam box with small air gaps and a fan system to improve the temperature homogeneity. Experimentally, the system was tested on stable temperature for 5 days achieving 94.4\% CE with 0.1\% RMS fluctuations (see Fig.9) and on ramping temperature for 6 days achieving $94.1 \% \mathrm{CE}$ with 1.36\% RMS fluctuations (see Fig.10). The correlation between CE and temperature on the breadboard surface can be seen in Fig.11. A strong positive correlation is observed with a slope of $0.33 \% / \mathrm{K}$. 
a)
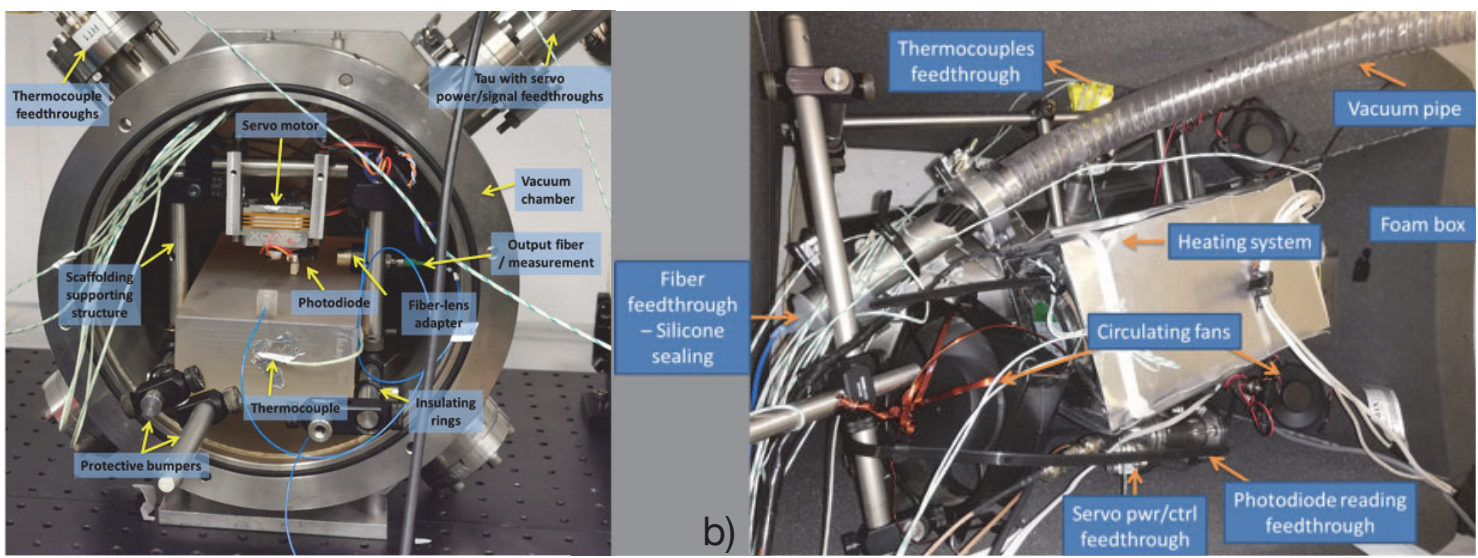

Figure 8: a) Final assembly of the vacuum system with the breadboard - I/O light measurement system and the vacuum feedthrough connectors, b) thermal enclosure
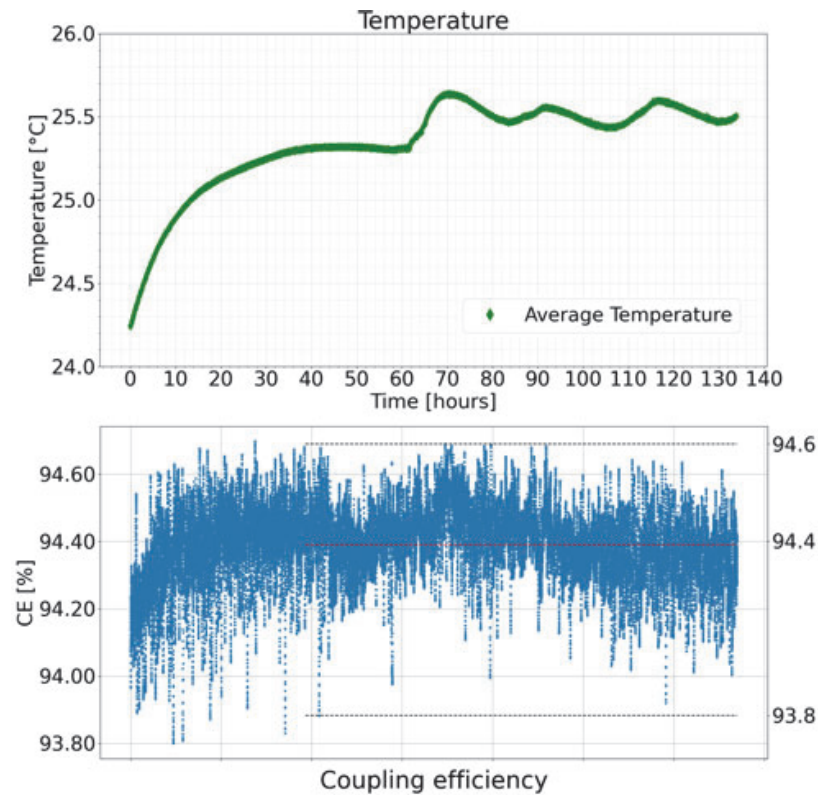

Figure 9: Thermal testing results for stable temperature: The system was initially cooled until the temperature on the walls of the vacuum reached a temperature of $11^{\circ} \mathrm{C}$ ) and then left to stabilize at room temperature for about 160 hours (considered stable for the last 120 hours). The max and min CE values is shown with black dashed while the red dashed line represents the mean CE value.

The performance of the prototype has been thoroughly tested in the presence of vibrations and complied with the requirements of a number of standards. The vibration testing was performed using RMS electrodynamic vibration test system: Shaker SW 6505 with a peak force of $11772 \mathrm{~N}$, max. acceleration $981 \mathrm{~m} / \mathrm{s} 2$, max. speed $1.20 \mathrm{~m} / \mathrm{s}$ and max displacement $13 \mathrm{~mm}$.

The tests were performed in three axes, including a potential mounting scheme see Fig. 12. The specifications for each axis were the following:

- Low-level survey (about $0.1 \mathrm{~g}$, swept up to $2000 \mathrm{~Hz}$ ) which constitutes a reference test allowing unexpected frequency shifts or amplification changes to be detected. The sweep was performed in 4 octaves $/ \mathrm{min}$. 


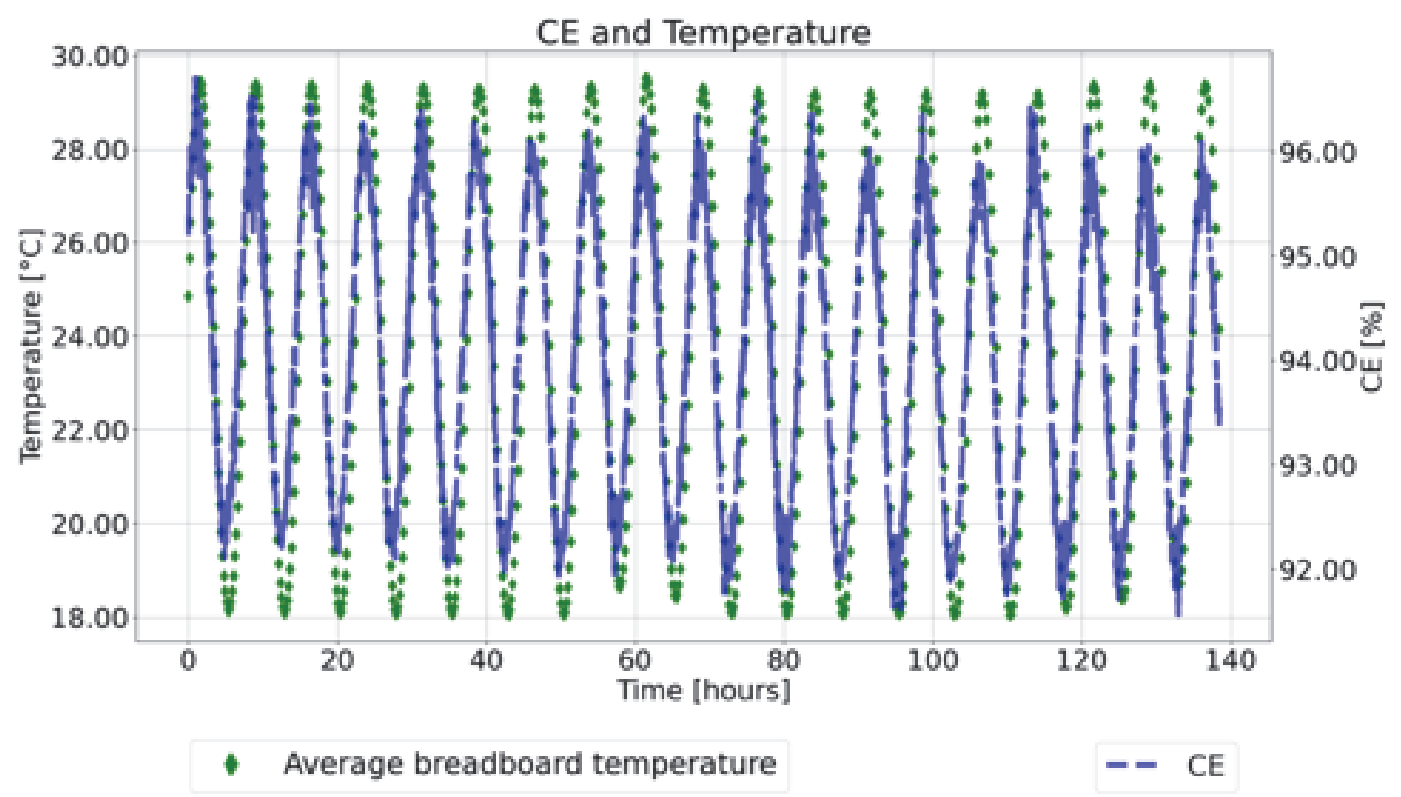

Figure 10: Thermal testing results for temperature cycling. Two axes are displayed, one for temperature and one for $\mathrm{CE}$.



Figure 11: Coupling efficiency as a function of the mean temperature of the breadboard for the same 140 hour run presented in fig. 10.

- Intermediate level sweep ( $-3 \mathrm{~dB}$ referenced to $3.5 \mathrm{~g}$, swept in range $5 \mathrm{~Hz} \rightarrow 100 \mathrm{~Hz} \rightarrow 5 \mathrm{~Hz}$ ); The sweep was performed in 4 octaves/min.

- Sweep in range $5 \rightarrow 100 \rightarrow 5 \mathrm{~Hz}$ at $3.5 \mathrm{~g}$. The sweep was performed in 4 octaves $/ \mathrm{min}$.

- Max 8.3 g RMS at a random spectrum between $20-2000 \mathrm{~Hz}$.

During the testing the operational performance of the breadboard was validated by measuring the power at the output fiber. Standard deviation at the order of one percent has been observed in the output power during the testing. After every test the $\mathrm{CE}$ was measured and no loss of power was observed within our measurement 


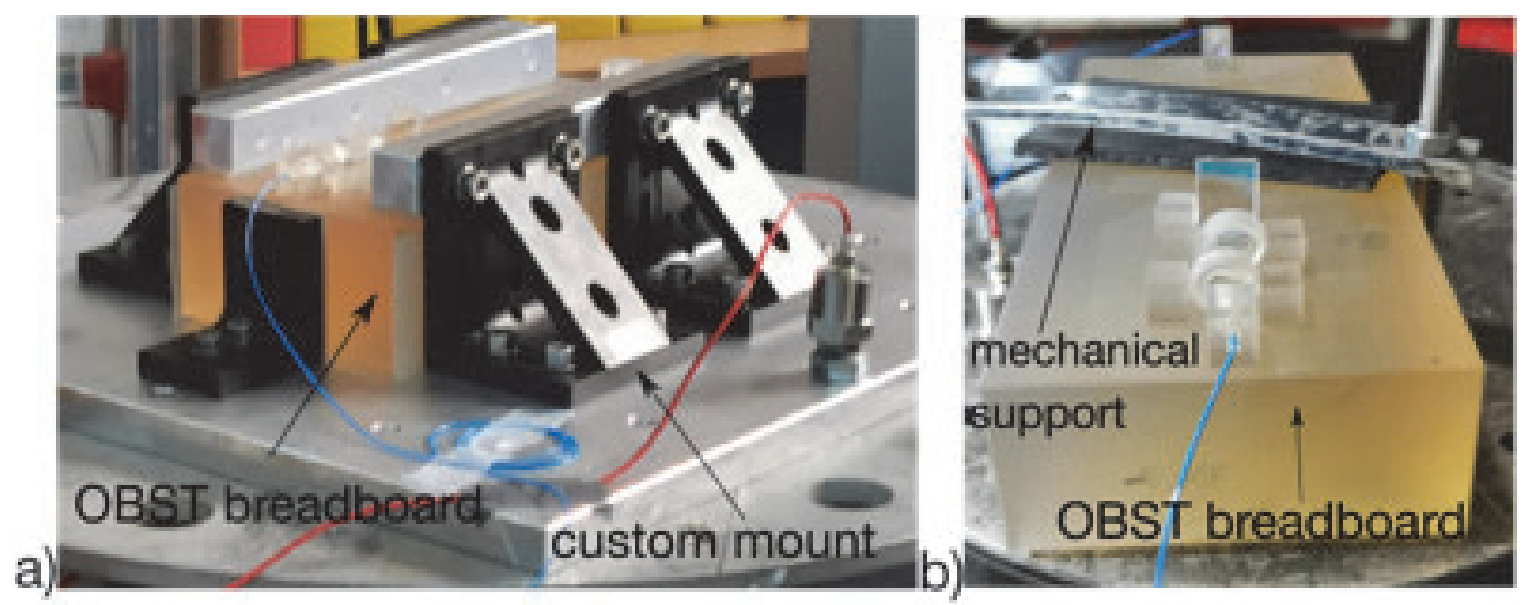

Figure 12: a) Mounting scheme of OBST designed mount plus adapter to the vibration table, b) bare mounting on the table using supports.

relative error which was at the order of $2 \%$. At the end of the test no visual damage, cracks, opening or loose parts were observed. The mounting technique of the breadboard has also been tested against a sinusoidal sweep of $3.5 \mathrm{~g}$ between 5 and $100 \mathrm{~Hz}$ and exhibited $0.15 \%$ RMS fluctuation in the output power. This shows the extreme stability of the breadboard, which not only survived an even harsher environment relative to standards for fiber optic components in space systems, but also exhibited the same behaviour over the duration of the tests. This can be seen in Fig. 13, where in the upper panel we plot the acceleration of the specimen while in the lower the CE. No significant change in the stability of the CE was observed in the presence of vibration. OBST has proven to not suffer any measurable plastic deformation as a result of the vibration testing (change in $C E<2 \%$ limited by measurement precision). The CE of OBST has also been tested with random accelerations at $8.2 \mathrm{~g}$ between $20 \mathrm{~Hz}$ and $2 \mathrm{kHz}$ with the RMS of fluctuations in $\mathrm{CE}$ being $0.28 \%$. The mounting scheme was also tested during a sinusoidal $3.5 \mathrm{~g}$ sweep test in a frequency range from $5 \mathrm{~Hz}$ to $100 \mathrm{~Hz}$ and found to comply to the relative standards.



Figure 13: Qualification level sweep: $0 \mathrm{~dB}, 3.5 \mathrm{~g}, 5 \rightarrow 100 \rightarrow 5 \mathrm{~Hz}$. CE remains stabilized at 94.8\%. CE $R M S=0.15 \%$. The upper panel shows the acceleration $[\mathrm{g}]$, the lower one the coupling efficiency [\%]. 


\section{SUMMARY AND OUTLOOK}

The main objective of this work was to build upon the basic principles and the knowledge gained during our previous work ${ }^{10}$ and optimize the design in order to meet the highly demanding needs of complex space missions. We have successfully demonstrated reduced system complexity, which led to reduced component manufacturing specification demands and offered alignment flexibility, stability and accuracy. In addition, the design was miniaturized by a factor of two relative to our previous work in volume increasing the compactness of our approach. Coupling efficiency at the order of $94 \%$ with $0.1 \%$ RMS variation under stable temperature and $1.36 \%$ during thermal cycling have been demonstrated. The vibration test campaign revealed that the breadboard complied to a number of standards (see Table 1) with the acceleration reaching $3.5 \mathrm{~g}$ in sweep mode, $8.3 \mathrm{~g}$ in random accelerations and $89.4 \mathrm{~g}$ for $1 \mathrm{~ms}$ in shock testing.

The proposed technology has potential applications in the context of earth observation. The long-term future in gravity sensing can benefit by the developments in atom interferometry, which could be considered in the context of the Copernicus Program. ${ }^{12}$ Just like in most potential cold atom missions, this results in rather extreme requirements in laser light conditioning that can be met with the use of OBST. In addition, any future mission using cold atoms in space, such as atom-interferometers, atom-quantum gravity missions etc could benefit from the proposed work. This include missions aiming to perform experiments in the context of dark matter search and gravitational-wave astronomy $\left(\mathrm{AEDGE}^{3}\right)$, gravity sensing and fundamental physics (STE-QUEST, ${ }^{5}$ $\left.\mathrm{MAQRO}^{4}\right)$. Furthermore any mission, which requires complex manipulation of light using active optics, such as quantum cryptography, can profit from OBST. Finally the proposed technology can find application in optical communication concepts where coupling incoming light into single mode fibers is essential.

Our future goal is to build upon the knowledge gained through OBST and proceed to the construction of a full light-conditioning module, including active optical elements (acousto-optical modulators, shutters), that has potential application in future cold-atom experiments in space.

\section{ACKNOWLEDGMENTS}

This work was supported by the European Space Agency (ESA) under contract No. 4000127622/19/NL/BJ/va, the Hellenic Foundation for Research and Innovation (HFRI) under Grant agreement 4794, and the General Secretariat for Research and Technology (GSRT).

\section{REFERENCES}

[1] Toyoshima, M., "Trends in satellite communications and the role of optical free-spacecommunications (invited)," J. Opt. Netw. 4, 300-311 (Jun 2005).

[2] Takenaka, H., Toyoshima, M., and Takayama, Y., "Experimental verification of fiber-coupling efficiency for satellite-to-ground atmospheric laser downlinks," Opt. Express 20, 15301-15308 (Jul 2012).

[3] El-Neaj, Y. A., Alpigiani, C., Amairi-Pyka, S., Araújo, H., Balaž, A., Bassi, A., Bathe-Peters, L., Battelier, B., Belić, A., Bentine, E., Bernabeu, J., Bertoldi, A., Bingham, R., Blas, D., Bolpasi, V., Bongs, K., Bose, S., Bouyer, P., Bowcock, T., Bowden, W., Buchmueller, O., Burrage, C., Calmet, X., Canuel, B., Caramete, L.-I., Carroll, A., Cella, G., Charmandaris, V., Chattopadhyay, S., Chen, X., Chiofalo, M. L., Coleman, J., Cotter, J., Cui, Y., Derevianko, A., De Roeck, A., Djordjevic, G. S., Dornan, P., Doser, M., Drougkakis, I., Dunningham, J., Dutan, I., Easo, S., Elertas, G., Ellis, J., El Sawy, M., Fassi, F., Felea, D., Feng, C.-H., Flack, R., Foot, C., Fuentes, I., Gaaloul, N., Gauguet, A., Geiger, R., Gibson, V., Giudice, G., Goldwin, J., Grachov, O., Graham, P. W., Grasso, D., van der Grinten, M., Gündogan, M., Haehnelt, M. G., Harte, T., Hees, A., Hobson, R., Hogan, J., Holst, B., Holynski, M., Kasevich, M., Kavanagh, B. J., von Klitzing, W., Kovachy, T., Krikler, B., Krutzik, M., Lewicki, M., Lien, Y.-H., Liu, M., Luciano, G. G., Magnon, A., Mahmoud, M. A., Malik, S., McCabe, C., Mitchell, J., Pahl, J., Pal, D., Pandey, S., Papazoglou, D., Paternostro, M., Penning, B., Peters, A., Prevedelli, M., Puthiya-Veettil, V., Quenby, J., Rasel, E., Ravenhall, S., Ringwood, J., Roura, A., Sabulsky, D., Sameed, M., Sauer, B., Schäffer, S. A., Schiller, S., Schkolnik, V., Schlippert, D., Schubert, C., Sfar, H. R., Shayeghi, A., Shipsey, I., Signorini, C., Singh, Y., Soares-Santos, M., Sorrentino, F., Sumner, T., Tassis, K., Tentindo, S., Tino, G. M., Tinsley, J. N., Unwin, J., Valenzuela, T., Vasilakis, G., Vaskonen, V., Vogt, C., Webber-Date, A., Wenzlawski, A., Windpassinger, 
P., Woltmann, M., Yazgan, E., Zhan, M.-S., Zou, X., and Zupan, J., "AEDGE: Atomic Experiment for Dark Matter and Gravity Exploration in Space," EPJ Quantum Technology 7(1), 6 (2020).

[4] Kaltenbaek, R., Aspelmeyer, M., Barker, P. F., Bassi, A., Bateman, J., Bongs, K., Bose, S., Braxmaier, C., Brukner, Č., Christophe, B., Chwalla, M., Cohadon, P.-F., Cruise, A. M., Curceanu, C., Dholakia, K., Diósi, L., Döringshoff, K., Ertmer, W., Gieseler, J., Gürlebeck, N., Hechenblaikner, G., Heidmann, A., Herrmann, S., Hossenfelder, S., Johann, U., Kiesel, N., Kim, M., Lämmerzahl, C., Lambrecht, A., Mazilu, M., Milburn, G. J., Müller, H., Novotny, L., Paternostro, M., Peters, A., Pikovski, I., Pilan Zanoni, A., Rasel, E. M., Reynaud, S., Riedel, C. J., Rodrigues, M., Rondin, L., Roura, A., Schleich, W. P., Schmiedmayer, J., Schuldt, T., Schwab, K. C., Tajmar, M., Tino, G. M., Ulbricht, H., Ursin, R., and Vedral, V., "Macroscopic Quantum Resonators (MAQRO): 2015 update," EPJ Quantum Technology 3(1), 5 (2016).

[5] Aguilera, D., Ahlers, H., Battelier, B., Bawamia, A., Bertoldi, A., Bondarescu, R., Bongs, K., Bouyer, P., Braxmaier, C., Cacciapuoti, L., Chaloner, C., Chwalla, M., Ertmer, W., Franz, M., Gaaloul, N., Gehler, M., Gerardi, D., Gesa, L., Gürlebeck, N., Hartwig, J., Hauth, M., Hellmig, O., Herr, W., Herrmann, S., Heske, A., Hinton, A., Ireland, P., Jetzer, P., Johann, U., Krutzik, M., Kubelka, A., Lämmerzahl, C., Landragin, A., Lloro, I., Massonnet, D., Mateos, I., Milke, A., Nofrarias, M., Oswald, M., Peters, A., Posso-Trujillo, K., Rasel, E., Rocco, E., Roura, A., Rudolph, J., Schleich, W., Schubert, C., Schuldt, T., Seidel, S., Sengstock, K., Sopuerta, C. F., Sorrentino, F., Summers, D., Tino, G. M., Trenkel, C., Uzunoglu, N., von Klitzing, W., Walser, R., Wendrich, T., Wenzlawski, A., Weßels, P., Wicht, A., Wille, E., Williams, M., Windpassinger, P., and Zahzam, N., "Corrigendum: STE-QUEST - test of the universality of free fall using cold atom interferometry (2014 class. quantum grav . 31 115010)," Classical and Quantum Gravity 31, 159502 (jul 2014).

[6] Lévèque, T., Faure, B., Esnault, F. X., Delaroche, C., Massonnet, D., Grosjean, O., Buffe, F., Torresi, P., Bomer, T., Pichon, A., Béraud, P., Lelay, J. P., Thomin, S., and Laurent, P., "Pharao laser source flight model: Design and performances," Review of Scientific Instruments 86(3), 033104 (2015).

[7] Robertson, D. I., Fitzsimons, E. D., Killow, C. J., Perreur-Lloyd, M., Ward, H., Bryant, J., Cruise, A. M., Dixon, G., Hoyland, D., Smith, D., and Bogenstahl, J., "Construction and testing of the optical bench for LISA pathfinder," Classical and Quantum Gravity 30, 085006 (mar 2013).

[8] Ren, W., Sun, Y., Wang, B., Xia, W., Qu, Q., Xiang, J., Dong, Z., Lü, D., and Liu, L., "Highly reliable optical system for a rubidium space cold atom clock," Appl. Opt. 55, 3607-3614 (May 2016).

[9] Liu, L., Lü, D.-S., Chen, W.-B., Li, T., Qu, Q.-Z., Wang, B., Li, L., Ren, W., Dong, Z.-R., Zhao, J.-B., Xia, W.-B., Zhao, X., Ji, J.-W., Ye, M.-F., Sun, Y.-G., Yao, Y.-Y., Song, D., Liang, Z.-G., Hu, S.-J., Yu, D.-H., Hou, X., Shi, W., Zang, H.-G., Xiang, J.-F., Peng, X.-K., and Wang, Y.-Z., "In-orbit operation of an atomic clock based on laser-cooled 87Rb atoms," Nature Communications 9(1), 2760 (2018).

[10] Drougakis, G., Mavrakis, K. G., Pandey, S., Vasilakis, G., Poulios, K., Papazoglou, D. G., and von Klitzing, W., "Precise and robust optical beam steering for space optical instrumentation," CEAS Space Journal 11(4), 589-595 (2019).

[11] Mylonakis, M., Pandey, S., Mavrakis, K. G., Drougakis, G., Vasilakis, G., Papazoglou, D. G., and von Klitzing, W., "Simple precision measurements of optical beam sizes," Appl. Opt. 57, 9863-9867 (Nov 2018).

[12] Aschbacher, J., [ESA's Earth Observation Strategy and Copernicus], 81-86, Springer Singapore, Singapore (2017). 